\title{
Security and Privacy Problems and Countermeasures of Internet of Things Applications
}

\author{
Junlan Liü, a \\ ${ }^{1}$ Xi'an International University, Shaanxi, Xi'an,China, 710077 \\ ${ }^{\mathrm{a}}$ email,
}

Keywords: Internet Technology; Internet Technology; Internet of Things; Privacy; Security

\begin{abstract}
Internet industry in today's development is still in the process of growth, so some of the implicit security and privacy issues need to be resolved, such as technical security is one of the biggest problems. This article from the Internet of things security and privacy significance of the distinction between the existence of the security and privacy characteristics of the analysis, and put forward the relevant security and privacy protection measures.
\end{abstract}

\section{Introduction}

Internet of things is the computer, Internet technology since the development of a new generation of network technology, and in the Internet, network communications and radio frequency and other technologies under the influence of each other, the development of things has become very fast. However, the information and fields involved in the Internet of Things are very broad, including the national power grid, military, aerospace and other countries important areas of jurisdiction, so the existence of security risks become an urgent concern, but also the Internet of Things The development of a more serious binding.

\section{Identify the Internet of Things Security and Privacy}

Internet of Things Security. The safety of the problem by the number of individuals to divide, can be public safety and personal safety; and then from the object type to divide, can be privacy, property, health, life, life, production and several. Security issues involve the stability of the public and the individual in the daily routine of life, work, study and entertainment. For the Internet of things, it contains information and material two different spaces, but also the formation of "people, machines, things," the combination of the three information systems [1]. The definition of the security of the Internet of things has not yet formed a concept of norms. This will be summarized as the following:

The security of information space requires that the information collected and stored in the Internet of Things system should have the characteristics of availability, confidentiality, integrity and easy management and control so as to satisfy people's inquiry about public information and the confidentiality of personal information.

The physical space security requirements, refers to the operation of the Internet of Things, the operation of material space to be feasible and reliable, and to ensure that it has a reasonable management and control features.

The Internet of Things system includes several important operation links, such as communication, calculation and control, to ensure the safe operation of the three, and improve its cooperation ability, so as to provide safe guarantee for the safe operation of the Internet of Things.

Internet of Things Privacy. The concept of privacy refers to the daily life of citizens, do not want to let others know or know the secret, including letters, personal resume, thought, physical, private life, etc., in general, individuals do not want to let others know things and things The Because of the importance of privacy, and gradually formed a right to privacy, privacy is the first time in 1890, Warren and Brandeis two American jurists in their book "Harvard Law Review" expressed the concept of privacy. After that, people for privacy and privacy of the discussion and theory in practice have been expanded and in-depth [2]. With the continuous discussion of people, 
the formation of a more extensive understanding: privacy refers to the natural person in the daily life of private life and information to enjoy the legal protection, not by others illegal collection, intrusion, knowledge and use, public and other rights which includes communication information, physical privacy, privacy, etc., and for its protection measures include technical and legal protection. And the development of the Internet of things environment, its "intelligent object perception ability to warn people, the need for the privacy of which involved in strict protection, has become a must solve the problem. This will be the Internet of things on privacy and privacy the content is summarized as follows:

Confidentiality. Confidentiality requirements, in the context of the development of the Internet of Things, the citizens of their own privacy should also have a confidential power to prevent others from knowing or using illegal. In addition, the Internet of private information collected by the Internet of Things, the owner of the privacy of the information should belong to the owner of the information, and for the Internet of personal information collection agencies, only possession of this part of the information, without the permission of the parties or the law Of circumstances, shall not be open or other illegal use.

Right to know. That is, when the collection of personal information in the collection of personal information or collected personal information, the owner of the information have the right to know their own information which is collected by the Internet of things, and the right to its information collection agencies to request inquiries. Internet of Things information agencies in the information is not legal provisions cannot inform the right person, are not allowed to refuse the right of the request.

Options. Internet of things in the collection of personal information before the need for access to the right person to be collected. In addition, the right holder has the right to choose their own information by which information network collection and collection of information collection agencies, and the use of information has the power to choose.

Domination. The right of the right holder to deal with the personal information contained therein shall, within the limits permitted by law, dispose of these privacy according to his own wishes, and shall, in accordance with his / her own interests, Your own will let others know or let others know.

Legal remedy. When the personal right of the privacy right has been illegally used, the right holder has the right to request the protection of the law and the right to claim compensation and remedy from the infringer.

Security claims. The right holder has the right to request the Internet of Things privacy information collection agencies to take their own information security to take appropriate protective measures, if the information collection agencies cannot provide the appropriate technology or measures, the right holder has the right to lift the information agency on their own private information collection rights.

Space privacy. Even the Internet of Things environment, but also to ensure that the private space of citizens cannot be disturbed, invaded, manipulated, snooping, etc. to ensure that citizens have their own personal privacy space.

\section{Features of Internet of Things Security and Privacy Issues}

Comprehensive Internet Security. The scale of the Internet continues to expand, so that the existence of network security issues is also very prominent. The security of the Internet of Things system and the local area network and the stand-alone system is quite different. The security affected by the stand-alone system can only be a single computer, and the security that the LAN can affect will be all the individuals in the whole LAN Privacy, the Internet will affect the security scope will be greater, may include the entire Internet of Things system operation, so the security threats facing the Internet of Things will be unprecedented [3].

Unrecoverable Operation. Internet of things to operate the object directly to the physical world, so the operation with the Internet is only for the simple data is very different. Internet security concerns are only information problems, and things with the perception, communication, interaction and data transmission and other functions, the operation of the entire physical environment. 
Therefore, the security threats faced by the Internet of Things include property, and even life safety, but also created a non-renewable operational characteristic of things.

Accurate and Comprehensive Privacy Perception. Relative to the Internet, the Internet of things more extensive processing equipment, which includes the sensor, positioning, camera, etc., can be the physical world of all people, things, things for accurate information records. Therefore, the comprehensive development of the Internet of Things will have a comprehensive and accurate access to personal life information, a great threat to personal privacy.

\section{Strategy of Security and Privacy of the Application of Internet of Things}

Technical Support. At present, although the application of things in life has become frequent, but most of the technology included in the growing stage, not yet fully mature, but also need further research and development. The entire Internet of Things system as all the technical integration, then each of the technology research and development and application need to consider the security and privacy protection. That is, the privacy protection, security, and manageability and control of the Internet of Things application need to be reflected in every detail and closely related to the application of each technology, which requires a long-term research and improvement [4]. This article will be one of the technical protection summarized as the following:

(1) Internet of Things architecture to have a higher degree of credibility. For the operation of the Internet of Things, its architecture is one of the support, but also one of the skeleton, the smooth use of other technologies, need to be based on the architecture.

(2) very secure data cloud mechanism. Internet of Things in the process of running will have a huge amount of data, and these data with each other between the larger or smaller differences.

(3) privacy data perception and processing technology. For the perception and processing of privacy data, should be user-centric, users have the right to their own wishes to regulate their own privacy can be known to others.

In short, the operation of the Internet of Things system is the operation of the technology, which need to ensure that the technology for the protection of personal privacy and security of which security.

Legal Safeguards. The development of Internet of things will make people's production, lifestyle changes have occurred, the current legal norms will be difficult to meet people's legal needs, so the development of things at the same time, the relevant laws and regulations are also an urgent need to constantly improve to maintain the synchronization with the development of things. The relevant laws should be concerned with the legal responsibility of the relevant "intelligent objects" in the application of the Internet of Things system, the legal norms of the collection, storage and use of information, and the identification and investigation of the criminal acts in the Internet of Things [5]. As the "smart object" in the operation of the Internet of Things has a certain degree of control in the information, so the responsibility of its behavior that need to make clear provisions. In addition, the application of Internet of things technology gradually wide, there may be a lot of criminals will use the Internet of things for criminal acts, and therefore also need to improve the risk of this part of the existence of the crime.

\section{Conclusion}

With the concept of things put forward, people's attention to the direction of development of things is also rising, but for the existence of security risks and privacy protection issues need to increase management. The development of things to the present, our side has been no less than the Internet of things connected to the "smart object", the most frequent is the monitoring, camera, and our cars, mobile phones and other commonly used positioning system are belong to the Internet of things a part of the system. And the Internet of information collected by the Internet are likely to involve a part of the natural person's privacy, then for its information collection process in the safety performance, should be recognized by the people. Followed by the storage conditions of these information should be collected security, the use of these information should be recognized by the 
rights of people, so as to protect the rights of the purpose of privacy. Internet security needs to be involved in a number of technologies, so you want to achieve the security of things and privacy protection, the safety performance of these technologies is the foundation; In addition, the relevant laws and regulations should also be based on the development of the Internet of things the existence of legal disputes to improve it is synchronized with the development of things.

\section{References}

[1] He Defeng, Sun Zhonghua. Internet of things in the development of security issues and countermeasures[J]. Electronic Technology, 2016, 04: 16-17 +12.

[2] Qiu Lei. Internet of things on the application of security and privacy issues [J]. Electronic Technology and Software Engineering, 2016, 12: 201.

[3] Lao Fei. Internet of things security issues and countermeasures[J]. Technology Information, 2014, 03: $11+18$.

[4] Li Juan. Internet security issues and countermeasures [J] .Computer CD-ROM software and applications, 2014,18: 47-48.

[5] Wang Zirui. On the security of things and related countermeasures[J]. Network Security Technology and Applications, 2017, 03: 170-171. 